\title{
A HYDROLOGIC VIEW ON BIOT'S THEORY OF POROELASTICITY
}

\author{
D. B. SILIN, V. A. KORNEEV, G. M. GOLOSHUBIN, AND T. W. PATZEK
}

\begin{abstract}
The main objective of this work is to obtain a simplified asymptotic representation of the reflection of seismic signal from a fluid-saturated porous medium in the low-frequency domain.

In the first part, we derive the equations of low-frequency harmonic waves in a fluid-saturated elastic porous medium from the basic concepts of filtration theory. We demonstrate that the obtained equations can be related to the poroelasticity model of Frenkel-Gassmann-Biot, and to pressure diffusion model routinely used in well test analysis as well. We thus try to put the poroelastic and filtration theories on the same ground.

We study the reflection of a low-frequency signal from a plane interface between elastic and elastic fluid-saturated porous media. We obtain an asymptotic scaling of the frequency-dependent component of the reflection coefficient with respect to a dimensionless parameter depending on the frequency of the signal and the reservoir fluid mobility. We also investigate the impact of the relaxation time and tortuosity on this scaling.
\end{abstract}

\section{INTRODUCTION}

When an acoustic wave crosses the boundary between elastic and fluid-saturated media, some energy of the wave is reflected and the rest is transmitted or dissipated. It is known that both the transmission and reflection coefficients from a fluid-saturated porous medium are functions of frequency $[18,15,35,12]$. Recently, low-frequency signals were successfully used in obtaining high-resolution images of oil and gas reservoirs [19, 20, 10] and in monitoring underground gas storage [23]. Therefore, understanding the behavior of the reflection coefficient at the low-frequency end of the seismic spectrum is of special importance.

In this work, we obtain a simplified asymptotic representation of the reflection of seismic signal from a fluid-saturated porous medium in the low-frequency domain. More specifically, we derive a relatively simple

Date: January 27, 2004.

Key words and phrases. Low-frequency signal, Darcy's law, seismic imaging. 
formula, where the frequency-dependent component of the reflection coefficient is proportional to the square root of the product of frequency of the signal and the mobility of the fluid in the reservoir. In our argument, we apply a somewhat nontraditional approach by deriving the wave propagation equations from the basic principles of the theory of filtration. This is done, in particular, to demonstrate that both the filtration and poroelasticity theories have much in common.

Indeed, both filtration theory $[29,31,6,3]$ and the theory of poroelasticity $[16,17,7,8,9,38]$ study, in particular, fluid flow in an elastic porous medium. The first theory usually assumes steady-state or transient processes where the macroscopic transition times are significantly larger than the transition times of the local microscopic processes. The second one, poroelasticity, includes a model of acoustic wave propagation in fluid-saturated elastic media, where the macroscopic transition times are small and, therefore, the concept of steady-state fluid flow may be inapplicable.

To obtain a system of equations characterizing fluid and solid interaction in a macroscopically homogeneous elastic fluid-saturated porous medium, we adopt the concept of relaxation filtration $[2,27,26]$, which is a modification of Darcy's law [11, 21, 22] in order to account for the inertial and non-equilibrium effects. We remind ourselves that originally Darcy's law was formulated for steady-state flow. It is recognized that non-equilibrium effects are important in two-phase flow [5, 4], see also [37]. However, due to local heterogeneities, they are important in single-phase flow as well.

Further, we demonstrate that under different assumptions, the equations obtained here can be transformed either into Biot's wave equations $[7,8,9]$, or into the elastic drive pressure diffusion equation [29, $25,3]$.

Originally, in the derivation of the wave equations of poroelasticity, the Hamiltonian principle of least action was applied [7, 8, 9]. In order to close the system, an introduction of a parameter having dimension of density was needed. This parameter was then related to a dimensionless tortuosity factor characterizing the complex geometry of the pore space in natural rocks. There are several definitions of tortuosity in the literature, see e.g., [6]. In Biot's derivation, the tortuosity factor statistically characterizes the heterogeneity of the local fluid velocity field [9]. The way this coefficient and the above-mentioned relaxation time enter the equations leads to the conclusion that both are linearly related to each other. The magnitude of the relaxation time, and thereby the value of the tortuosity as well, affects the way the reflection coefficient depends on the frequency. Since the magnitude of the tortuosity in 
Biot's equations ranges, in general, between one to infinity [28], it is very important to know typical tortuosity factors for different types of rock. Microscopic flow modeling on pore networks [30] and morphological pore geometry analysis of tomographic images of the pore space [36] are very promising approaches from this point of view.

During the last fifty years, a significant effort has been spent on the investigations of attenuation of Biot's waves, see e.g., $[33,34]$ and the references therein. It has been noticed that there must be a relation between the dependence of the attenuation on the wave frequency and the permeability of the reservoir [32]. In many cases, the attenuation coefficient can be obtained in an explicit, but quite cumbersome, form. Computation of the reflection coefficient is even more complex because it additionally requires inversion of a matrix, so even under simplifying assumptions the formula is cumbersome. In this study, we obtain a simplified asymptotic expression where the role of the reservoir fluid mobility is transparent. We focus on the simplest case of normal pwave reflection. In addition, we assume that the grains of the solid skeleton are practically incompressible, so that all deformations of the rock and the pore space are due to the rearrangements of the grains. A more general study will be reported later.

The layout of the paper is as follows. In the next section, we setup the model and derive main equations from the principles of filtration theory. In Section 3, we relate the obtained relationships to the Biot's equations and pressure diffusion or elastic drive model. Then, in the next section, we obtain a harmonic wave solution to the equations obtained in Section 2 and introduce the dimensionless small parameters for the asymptotic analysis. In the Section 6 , we obtain the asymptotic expression for the reflection coefficient. In Section 7, we elaborate on the role that the relaxation time and tortuosity play in our asymptotic analysis. We end the paper with conclusions and acknowledgments.

\section{FLUID-SOLID SKELETON INTERACTION EQUATIONS}

Let us consider a homogeneous porous medium $M$ whose pore space is filled with a viscous fluid. We consider a situation where the grains of the solid skeleton are displaced by an elastic wave. We assume that the wave is aligned with the $x$-axis of a fixed coordinate system. We consider a p-wave only. Thus, after averaging over a plane orthogonal to $x$, the only non-zero component of the displacement is the $x$-component. In other words, the mean displacement is one-dimensional. Due to the deformation of the skeleton, the grains are rearranged. We assume that the rearrangement occurs through elastic deformations of the cement 
bonds between the grains. Such an assumption is natural in many situations considered in hydrology and is quite common in the geophysical literature as well, see, e.g., [12].

In general, deformations result in energy dissipation. For simplicity, in this paper we assume that these energy losses are much smaller than the losses through the viscous friction in the cross-flow of the reservoir fluid. Further, we assume that the rock is "clean", so that the total mass and volume of the bonds is small relative to those of the grains. Thus, for the bulk density of the "dry" skeleton $\varrho$ we have

$$
\varrho=(1-\phi) \varrho_{g}
$$

where $\varrho_{g}$ is the density of the grains and $\phi$ is the porosity. Let us denote by $u$ the mean displacement of the skeleton grains in the direction $x$. If we neglect the microscopic rotational motions of the grains, the mean density of momentum of a drained skeleton is given by

$$
\varrho \frac{\partial u}{\partial t}=(1-\phi) \varrho_{g} \frac{\partial u}{\partial t}
$$

where $t$ denotes time.

Deformations of the skeleton change the stress field. It is natural to assume that the shear stresses are, on average, uniformly distributed in any direction orthogonal to $x$. In general, even uniformly distributed shear stress influences the rearrangement of the skeleton. However, our assumption of stiff grains and small-volume bonds makes it possible to neglect this influence. The $x$-component of the stress caused by a displacement $u, \sigma_{x}$, can be measured by the elastic forces acting on a unit (bulk) area in a plane orthogonal to $x$. Linear elasticity hypothesis suggests that for small displacements, $\sigma_{x}$ and $u$ are linearly related:

$$
\sigma_{x}=-\frac{1}{\beta_{x}} \frac{\partial u}{\partial x}
$$

Here $\beta_{x}$ is the respective uniaxial elastic coefficient. We retain index $x$ in equation (3) just to emphasize that here we focus our attention on a one-dimensional case only.

The motion of the reservoir fluid can be characterized by the superficial or Darcy velocity $W$ measured relative to the skeleton. This means, that if we imagine a small surface element moving along with the local displacement of the grains, then the volumetric fluid flux through this surface is equal to the projection of $W$ on the unit normal vector to the surface. The average velocity $v_{f}$ of the fluid relative to the skeleton is related to the Darcy velocity by equation

$$
\phi v_{f}=W
$$


We assume that both the motion of the skeleton $u$ and Darcy velocity $W$ are just small perturbations near some equilibrium values. The same applies to the fluid pressure $p$.

The fluid pressure gradient has a two-fold impact on the total momentum balance in the fluid-solid system [31,38]. First, it acts through the pressure buoyancy force applied to the fluid in a representative volume of the porous material. This force is equal to $-\phi \frac{\partial p}{\partial x}$. Second, since the fluid and the skeleton may move with different velocities, there is momentum transfer between the solid and the fluid through viscous friction at the pore walls. This second component is usually called seepage drag and is equal to $-(1-\phi) \frac{\partial p}{\partial x}$. Thus, the total fluid pressure-related force acting on the fluid-skeleton system is equal to $-\frac{\partial p}{\partial x}$. A small volume of the medium, $\delta V$, contains $\varrho \delta V$ mass of rock material and $\phi \varrho_{f} \delta V$ mass of fluid. Here $\varrho_{f}$ is the density of the fluid. Hence, the momentum of moving fluid per unit bulk volume is

$$
\phi \varrho_{f}\left(\frac{\partial u}{\partial t}+v_{f}\right)=\phi \varrho_{f} \frac{\partial u}{\partial t}+\varrho_{f} W
$$

Thus, for the total fluid-rock bulk momentum density, we obtain the following equation implied by Newton's second law:

$$
\varrho_{b} \frac{\partial^{2} u}{\partial t^{2}}+\varrho_{f} \frac{\partial W}{\partial t}=\frac{1}{\beta} \frac{\partial^{2} u}{\partial x^{2}}-\frac{\partial p}{\partial x}
$$

Here $\varrho_{b}$ is the bulk density of the fluid-saturated medium:

$$
\varrho_{b}=(1-\phi) \varrho_{g}+\phi \varrho_{f}=\varrho+\phi \varrho_{f}
$$

Now, let us consider in more detail the motion of the fluid. According to Darcy's law, at steady-state conditions,

$$
W=-\frac{\kappa}{\eta} \frac{\partial \Phi}{\partial x}
$$

where $\kappa$ is the permeability of the medium, $\eta$ is the viscosity of the fluid and $\Phi$ is the flow potential $[21,22]$. Let us recall that we consider only small perturbations near an equilibrium configuration and that the Darcy velocity $W$ is measured relative to the porous medium. Hence, the gravity term in the differential of potential $\Phi$ is replaced with a term characterizing additional pressure drop due the accelerated motion of the skeleton, i.e.,

$$
d \Phi=d p+\varrho_{f} \frac{\partial^{2} u}{\partial t^{2}} d x
$$


Darcy's law (8) is for steady-state flow. If the flow is transient, e.g., due to abrupt changes in the pressure field, equation (8) may need to be modified in order to account for inertial and non-equilibrium effects. As the pressure gradient changes, the local redistribution of the pressure field does not occur instantaneously because it includes microscopic fluid flow along and between the pores. Using dimensional considerations and linearization, we replace equation (8) with a nonequilibrium relationship

$$
W+\tau \frac{\partial W}{\partial t}=-\frac{\kappa}{\eta} \frac{\partial \Phi}{\partial x}
$$

Here $\tau$ is a characteristic redistribution time. Such a modification was proposed by Alishaev $[1,2]$. In multiphase flow, similar considerations were used to model non-equilibrium effects at the front of water-oil displacement and spontaneous imbibition $[5,4]$. Some results on estimation of the relaxation time based on interpretation of experiments were reported in $[27,26,13]$. Apparently, the relaxation time is a function of both pore space geometry and fluid viscosity $\eta$ and compressibility $\beta_{f}$. Dimensional considerations suggest that $\tau=\eta \beta_{f} F\left(\kappa / L^{2}\right)$, where $L$ is the characteristic size of an elementary representative volume of the medium and $F$ is some dimensionless function. Time $\tau$ is apparently related to the tortuosity factor [9]. This relationship is discussed in more detail below.

Thus, summing up, we arrive at the following equation characterizing the dynamics of the motion of the fluid

$$
W+\tau \frac{\partial W}{\partial t}=-\frac{\kappa}{\eta} \frac{\partial p}{\partial x}-\varrho_{f} \frac{\kappa}{\eta} \frac{\partial^{2} u}{\partial t^{2}}
$$

The mass conservation for the fluid and the solid skeleton imply

$$
\begin{aligned}
\frac{\partial \varrho_{f} \phi}{\partial t} & =-\frac{\partial\left(\varrho_{f} W+\phi \varrho_{f} \frac{\partial u}{\partial t}\right)}{\partial x} \\
\frac{\partial \varrho}{\partial t} & =-\frac{\partial}{\partial x}\left(\varrho \frac{\partial u}{\partial t}\right)
\end{aligned}
$$

For the fluid, we apply the adiabatic compressibility law [24], that is, for small fluid pressure perturbation

$$
\frac{d \varrho_{f}}{\varrho_{f}}=\beta_{f} d p
$$


Hence, Eq. (12) can be rewritten as

$$
\frac{\partial \phi}{\partial t}+\phi \beta_{f} \frac{\partial p}{\partial t}=-\frac{\partial W}{\partial x}-\phi \frac{\partial^{2} u}{\partial x \partial t}-W \frac{\partial \varrho_{f}}{\partial x}-\frac{1}{\varrho_{f}} \frac{\partial}{\partial x}\left(\phi \varrho_{f}\right) \frac{\partial u}{\partial t}
$$

Due to the smallness of the perturbations, the last two terms in equation (15) are small of a higher order and can be neglected.

By virtue of equation (1), equation (13) takes on the form

$$
-\frac{\partial \phi}{\partial t}+(1-\phi) \frac{1}{\varrho_{g}} \frac{\partial \varrho_{g}}{\partial t}=-\frac{1}{\varrho_{g}}(1-\phi) \frac{\partial \varrho_{g}}{\partial x} \frac{\partial u}{\partial t}+\frac{\partial \phi}{\partial x} \frac{\partial u}{\partial t}-(1-\phi) \frac{\partial^{2} u}{\partial x \partial t}
$$

Again, the smallness of the perturbations implies that the first two terms on the right-hand side of the last equation can be dropped. Further on, perturbation of the grain density is a linear function of the perturbations of the stress and fluid pressure, that is

$$
\frac{1}{\varrho_{g}} d \varrho_{g}=\beta_{g s} d \sigma_{x}+\beta_{g f} d p
$$

where $\beta_{g s}$ and $\beta_{g f}$ are the respective compressibility coefficients. Thus, equation (16) can be written as

$$
\frac{\partial \phi}{\partial t}=(1-\phi) \beta_{g f} \frac{\partial p}{\partial t}+(1-\phi)\left(1+\frac{\beta_{g s}}{\beta}\right) \frac{\partial^{2} u}{\partial x \partial t}
$$

A combination of this last result with equation (15) leads to the following relationship

$$
\left(1+(1-\phi) \frac{\beta_{g s}}{\beta}\right) \frac{\partial^{2} u}{\partial x \partial t}+\left(\phi \beta_{f}+(1-\phi) \beta_{g f}\right) \frac{\partial p}{\partial t}=-\frac{\partial W}{\partial x}
$$

Under the assumptions formulated above, the compressibility of the grains is much smaller than the compressibility of the fluid and the skeleton:

$$
\beta_{g f} \ll \beta_{f} \quad \text { and } \quad \beta_{g s} \ll \beta
$$

This means that the deformation manifests itself only through the porosity perturbations only. In such a case, equation (19) can be further reduced to

$$
\frac{\partial^{2} u}{\partial x \partial t}+\phi \beta_{f} \frac{\partial p}{\partial t}=-\frac{\partial W}{\partial x}
$$

Note that there is no qualitative difference between equations (19) and (21). To summarize, we have obtained a closed system of three equations (6), (11), and (19) with three unknown functions of $t$ and $x$ : skeleton displacement $u$, fluid pressure $p$, and Darcy velocity $W$. 


\section{Relationship to Biot's POROElasticity AND PRESSURE DIFFUSION EQUATIONS}

In this section, we demonstrate that under the assumptions formulated in Section 2 equations (6), (11), and (19) can be reduced to the system of equations obtained by Biot [7, 9], see also [14]. At the same time, neglecting the inertial terms in these equations, leads to the pressure diffusion equation used in hydrology and petroleum engineering for well test analysis, see $[25,3]$.

We begin with Biot's theory. We will perform the calculations using the assumption (20). As we consider only small oscillatory deformations of the skeleton and fluctuations of the fluid flow, we can introduce a "superficial" displacement of the fluid relative to the skeleton $w$, so that

$$
W=\frac{\partial w}{\partial t}
$$

Note that inasmuch as $w$ is related by Eq. (22) to the Darcy velocity of the fluid, it is different from the average microscopic fluid displacement. Substitution of (22) into equation (21) yields

$$
\frac{\partial^{2} u}{\partial x \partial t}+\phi \beta_{f} \frac{\partial p}{\partial t}=-\frac{\partial^{2} w}{\partial t \partial x}
$$

By integration in $t$ and differentiation in $x$, we obtain

$$
\frac{\partial p}{\partial x}=-\frac{1}{\phi \beta_{f}} \frac{\partial^{2} u}{\partial x^{2}}-\frac{1}{\phi \beta_{f}} \frac{\partial^{2} w}{\partial x^{2}}
$$

Here we have utilized the assumption of the smallness of the rock-fluid system oscillations near an equilibrium configuration. Otherwise, due to the integration, equation (24) should include an unknown function of $x$. Now, let us substitute (22) and the result (24) in equations (6) and (11):

$$
\begin{aligned}
\varrho_{b} \frac{\partial^{2} u}{\partial t^{2}}+\varrho_{f} \frac{\partial^{2} w}{\partial t^{2}} & =\left(\frac{1}{\beta}+\frac{1}{\phi \beta_{f}}\right) \frac{\partial^{2} u}{\partial x^{2}}+\frac{1}{\phi \beta_{f}} \frac{\partial^{2} w}{\partial x^{2}} \\
\varrho_{f} \frac{\partial^{2} u}{\partial t^{2}}+\tau \frac{\eta}{\kappa} \frac{\partial^{2} w}{\partial t^{2}} & =\frac{1}{\phi \beta_{f}} \frac{\partial^{2} u}{\partial x^{2}}+\frac{1}{\phi \beta_{f}} \frac{\partial^{2} w}{\partial x^{2}}-\frac{\eta}{\kappa} \frac{\partial w}{\partial t}
\end{aligned}
$$

Under the assumptions formulated above, equations (25) - (26) are equivalent to the Biot system of equations [7, 9]. Comparing the individual terms, we can establish a relationship between the relaxation time and the tortuosity factor. Namely, we infer that the relaxation time $\tau$ is related to the dynamic coupling coefficient $m[9]$ through the mobility ratio $\kappa / \eta$. The dynamic coupling coefficient is often expressed 
through the tortuosity factor $T: m=T \varrho_{f} / \phi$. Hence, for the tortuosity and relaxation time, we obtain the following relationship

$$
T=\tau \frac{\eta \phi}{\kappa \varrho_{f}} \quad \text { or } \quad \tau=T \frac{\kappa \varrho_{f}}{\eta \phi}
$$

Further comparison between the elastic coefficients reveals that under the assumptions (20) the coefficient $\gamma$ in the notations of [14] is equal to one and the other coefficients are, respectively, equal

$$
H=\frac{1}{\beta}+\frac{1}{\phi \beta_{f}} \quad \text { and } \quad 2 D=\frac{1}{\phi \beta_{f}}
$$

Now, let us derive the pressure diffusion equation. Assume that the characteristic time $t_{D}$ of the process is large in comparison with the relaxation time $\tau$ and the displacements of the skeleton are much smaller then the characteristic length scale of the process $L$ :

$$
t_{D} \gg \tau \quad \text { and } \quad u \ll L
$$

Under this assumption, we can drop the second order time derivatives of displacement $u$ and time derivatives of Darcy velocity $W$ in equations (6) and (11):

$$
\begin{aligned}
\frac{\partial p}{\partial x} & =\frac{1}{\beta} \frac{\partial^{2} u}{\partial x^{2}} \\
W & =-\frac{\kappa}{\eta} \frac{\partial p}{\partial x}
\end{aligned}
$$

By integrating equation (30) in $x$ and differentiating in $t$ yields

$$
\frac{\partial^{2} u}{\partial t \partial x}=\beta \frac{\partial p}{\partial t}
$$

Formally, integration by $x$ is defined up to a function of time. Assuming a constant pressure at infinity, this function of time is, in fact, constant. This constant is then cancelled by the differentiation with respect to t. Finally, by a substitution of equations (31) and (32) into (19), we obtain

$$
\phi\left(\beta / \phi+\beta_{f}\right) \frac{\partial p}{\partial t}=\frac{\kappa}{\eta} \frac{\partial^{2} p}{\partial x^{2}}
$$

This last equation is the pressure diffusion equation routinely used in well test analysis $[25,3]$. 


\section{Plane Compression WAVE: an Asymptotic SOlUtion}

Let us consider the system of equations obtained in Section 2. We introduce a dimensionless pressure

$$
P=\phi \beta_{f} p
$$

and the hydraulic diffusivity

$$
D=\frac{\kappa}{\phi \beta_{f} \eta}
$$

Dividing equation (6) by $\varrho_{b}$ and putting

$$
v_{b}^{2}=\frac{1}{\beta \varrho_{b}} \quad \text { and } \quad v_{f}^{2}=\frac{1}{\phi \beta_{f} \varrho_{b}}
$$

we obtain

$$
\begin{aligned}
\frac{\partial^{2} u}{\partial t^{2}}+\frac{\varrho_{f}}{\varrho_{b}} \frac{\partial W}{\partial t} & =v_{b}^{2} \frac{\partial^{2} u}{\partial x^{2}}-v_{f}^{2} \frac{\partial P}{\partial x} \\
\lambda_{f} \frac{\partial^{2} u}{\partial t^{2}}+W+\tau \frac{\partial W}{\partial t} & =-D \frac{\partial P}{\partial x} \\
\gamma_{1} \frac{\partial^{2} u}{\partial x \partial t}+\gamma_{2} \frac{\partial P}{\partial t} & =-\frac{\partial W}{\partial x}
\end{aligned}
$$

where

$$
\lambda_{f}=\varrho_{f} \frac{\kappa}{\eta}
$$

is the "kinematic" mobility of the fluid, and

$$
\gamma_{1}=1+(1-\phi) \frac{\beta_{g s}}{\beta} \quad \text { and } \quad \gamma_{2}=1+(1-\phi) \frac{\beta_{g f}}{\phi \beta_{f}}
$$

Clearly, $\lambda_{f}$ has the dimension of time. We seek a planar wave solution to the equations (37)-(39), i.e., we look for a solution in the form

$$
u=U_{s} e^{i(\omega t-k x)}, \quad W=W_{f} e^{i(\omega t-k x)}, \quad \text { and } \quad P=P_{0} e^{i(\omega t-k x)}
$$

Substitution of Eq. (42) into (37)-(39) produces a system of algebraic equations

$$
\left\{\begin{aligned}
-\omega^{2} U_{s}+i \omega \frac{\varrho_{f}}{\varrho_{b}} W_{f} & =-v_{b}^{2} k^{2} U_{s}+i v_{f}^{2} k P_{0} \\
-\lambda_{f} \omega^{2} U_{s}+i \tau \omega W_{f} & =i D k P_{0}-W_{f} \\
k \omega \gamma_{1} U_{s}+i \omega \gamma_{2} P_{0} & =i k W_{f}
\end{aligned}\right.
$$

Then using the last equation, $W_{f}$ can be eliminated from the system (43). Indeed, we get

$$
W_{f}=-i \omega \gamma_{1} U_{s}+\omega \gamma_{2} \frac{P_{0}}{k}
$$


and

$$
i \omega W_{f}=\omega^{2} \gamma_{1} U_{s}+i \omega^{2} \gamma_{2} \frac{P_{0}}{k}
$$

Then,

$$
\left\{\begin{aligned}
-\omega^{2}\left(1-\frac{\varrho_{f}}{\varrho_{b}} \gamma_{1}\right) U_{s}+i \omega^{2} \gamma_{2} \frac{\varrho_{f}}{\varrho_{b}} \frac{P_{0}}{k} & =-v_{b}^{2} k^{2} U_{s}+i v_{f}^{2} k P_{0} \\
-\left(\lambda_{f}-\tau \gamma_{1}\right) \omega^{2} U_{s}+i \tau \omega^{2} \gamma_{2} \frac{P_{0}}{k} & =i D k P_{0}+i \omega \gamma_{1} U_{s}-\omega \gamma_{2} \frac{P_{0}}{k}
\end{aligned}\right.
$$

Now, let us introduce two new variables

$$
v=\frac{\omega}{k} \quad \text { and } \quad \zeta=-\frac{i P_{0}}{k U_{s}}
$$

Note, that $v$ has the dimension of velocity and, in general, $v$ is a complex quantity. The variable $\zeta$ and equation (44) relate all three amplitudes $U_{s}, W_{f}$, and $P_{0}$. In particular,

$$
W_{f}=i \omega\left(-\gamma_{1}+\gamma_{2} \zeta\right) U_{s}=\frac{\omega}{k}\left(-\frac{\gamma_{1}}{\zeta}+\gamma_{2}\right) P_{0}
$$

In terms of the "velocity" $v$ and $\zeta$, the system of equations (46) takes on the following form

$$
\left\{\begin{aligned}
v^{2}\left(1-\gamma_{1} \frac{\varrho_{f}}{\varrho_{b}}\right)+v^{2} \gamma_{2} \frac{\varrho_{f}}{\varrho_{b}} \zeta & =v_{b}^{2}+v_{f}^{2} \zeta \\
\left(\lambda_{f}-\tau \gamma_{1}\right) v^{2}+\tau v^{2} \gamma_{2} \zeta & =D \zeta+\frac{i}{\omega} v^{2}\left(-\gamma_{1}+\gamma_{2} \zeta\right)
\end{aligned}\right.
$$

or, equivalently,

$$
\left\{\begin{aligned}
v^{2}\left(1-\gamma_{1} \frac{\varrho_{f}}{\varrho_{b}}+\zeta \gamma_{2} \frac{\varrho_{f}}{\varrho_{b}}\right) & =v_{b}^{2}+v_{f}^{2} \zeta \\
v^{2}\left[\lambda_{f}-\left(\tau-\frac{i}{\omega}\right)\left(\gamma_{1}-\gamma_{2} \zeta\right)\right] & =D \zeta
\end{aligned}\right.
$$

Denote

$$
\tau_{D}=\frac{D}{v_{f}^{2}}=\frac{\kappa \varrho_{b}}{\eta}, \quad \gamma_{v}=\frac{v_{b}^{2}}{v_{f}^{2}}=\frac{\phi \beta_{f}}{\beta} \quad \text { and } \quad \gamma_{\varrho}=\frac{\varrho_{f}}{\varrho_{b}}
$$

The parameters $\gamma_{v}$ and $\gamma_{\varrho}$ are dimensionless. From equation (40),

$$
\lambda_{f}=\gamma_{\varrho} \tau_{D}
$$

In addition, let us define dimensionless relaxation time and angular frequency

$$
\theta=\frac{\tau}{\tau_{D}} \quad \text { and } \quad \varepsilon=\tau_{D} \omega
$$


After all these preparations, let us divide equations (50) by $v_{f}^{2}$ and put $V=\frac{v}{v_{f}}$. We obtain

$$
\left\{\begin{aligned}
V^{2}\left(1-\gamma_{1} \gamma_{\varrho}+\zeta \gamma_{2} \gamma_{\varrho}\right) & =\gamma_{v}+\zeta \\
V^{2}\left[\gamma_{\varrho}-\left(\theta-\frac{i}{\varepsilon}\right)\left(\gamma_{1}-\gamma_{2} \zeta\right)\right] & =\zeta
\end{aligned}\right.
$$

By eliminating $V$,

$$
V^{2}=\frac{\gamma_{v}+\zeta}{1-\gamma_{1} \gamma_{\varrho}+\gamma_{2} \gamma_{\varrho} \zeta}
$$

we obtain a quadratic equation with respect to $\zeta$ :

$$
\begin{array}{r}
\left(\gamma_{2}+i \varepsilon\left(-\gamma_{2} \gamma_{\varrho}+\theta \gamma_{2}\right)\right) \zeta^{2} \\
+\left(-\gamma_{1}+\gamma_{2} \gamma_{v}+i \varepsilon\left[-1+\gamma_{1} \gamma_{\varrho}+\left(\gamma_{\varrho}-\theta \gamma_{1}\right)+\theta \gamma_{2} \gamma_{v}\right]\right) \zeta \\
+\left(-\gamma_{1} \gamma_{v}+i \varepsilon \gamma_{v}\left(\gamma_{\varrho}-\tau \gamma_{1}\right)\right)=0
\end{array}
$$

At $\varepsilon=0$, equation (56) reduces to

$$
\gamma_{2} \zeta^{2}+\left(-\gamma_{1}+\gamma_{2} \gamma_{v}\right) \zeta-\gamma_{1} \gamma_{v}=0
$$

The latter equation admits two real roots

$$
\zeta_{0}^{(1)}=-\gamma_{v} \quad \text { and } \quad \zeta_{0}^{(2)}=\frac{\gamma_{1}}{\gamma_{2}}
$$

By virtue of (20) and (41), the absolute value of the first root $\zeta_{0}^{1}$ is close to unity, whereas the absolute value of the second one is equal to $\frac{\phi \beta_{f}}{\beta}$, that is in many cases larger than one. Using equation (55), we obtain two asymptotic values for the "velocity" $v$

$$
v_{0}^{(1)}=0 \quad \text { and } \quad v_{0}^{(2)}=v_{f} \sqrt{\gamma_{v}+\frac{\gamma_{1}}{\gamma_{2}}}
$$

That means that the first solution corresponds to the slow wave, whereas the second one is related to the fast wave.

The exact solution to Eq. (56) can be easily obtained, but the expression is quite cumbersome. Instead, let us look for an asymptotic solution. Note that if we assume the permeability $\kappa \sim 1$ Darcy, that is $\kappa \sim 10^{-12} \mathrm{~m}^{2}$, the viscosity of the fluid $\eta \sim 1 \mathrm{cP}=10^{-3} \mathrm{~Pa}-\mathrm{s}$, and the bulk density of the rock $\varrho_{b} \sim 10^{3} \mathrm{~kg} / \mathrm{m}^{3}$, then $\varepsilon \preceq 10^{-3}$ for frequencies $\omega$ not exceeding the order of $1 \mathrm{kHz}$. Inasmuch as $\gamma_{1}$ and $\gamma_{2}$ are of the order of unity, we can assume that $\varepsilon$ is a small parameter in equation (56) and we can look for a solution in the form

$$
\zeta=\zeta_{0}+\zeta_{1} i \varepsilon-\zeta_{2} \varepsilon^{2} \ldots
$$


For brevity, let us put

$$
\begin{array}{lll}
A_{0}=\gamma_{2} & A_{1}=-\gamma_{2} \gamma_{\varrho}+\theta \gamma_{2} \\
B_{0}=\gamma_{2} \gamma_{v}-\gamma_{1} & B_{1}=-1+\gamma_{\varrho}\left(1+\gamma_{1}\right)+\theta\left(\gamma_{2} \gamma_{v}-\gamma_{1}\right) \\
C_{0}=-\gamma_{1} \gamma_{v} & C_{1}=\gamma_{v}\left(\gamma_{\varrho}-\theta \gamma_{1}\right)
\end{array}
$$

Then,

$$
\zeta_{1}=-\frac{A_{1} \zeta_{0}^{2}+B_{1} \zeta_{0}+C_{1}}{2 A_{0} \zeta_{0}+B_{0}}
$$

Thus, for the slow and fast waves we respectively get

$$
\zeta_{1}^{(1)}=\gamma_{v} \frac{1-\gamma_{\varrho}\left(\gamma_{2} \gamma_{v}+\gamma_{1}\right)}{\gamma_{1}+\gamma_{2} \gamma_{v}}
$$

and

$$
\zeta_{1}^{(2)}=\frac{1}{\gamma_{2}} \frac{\gamma_{1}-\gamma_{\varrho}\left(\gamma_{2} \gamma_{v}+\gamma_{1}\right)}{\gamma_{1}+\gamma_{2} \gamma_{v}}
$$

Note, that since both are almost equal to one, $\gamma_{1} \approx 1$ and $\gamma_{2} \approx 1$ equations (63) and (64) can be simplified

$$
\begin{aligned}
\zeta_{1}^{(1)} & =\gamma_{v} \frac{1-\gamma_{\varrho} \gamma_{v}-\gamma_{\varrho}}{1+\gamma_{v}} \\
\zeta_{1}^{(2)} & =\frac{1}{\gamma_{2}} \frac{\gamma_{1}-\gamma_{\varrho} \gamma_{v}-\gamma_{\varrho}}{1+\gamma_{v}}
\end{aligned}
$$

In particular, $\zeta_{1}^{(1)}$ and $\zeta_{1}^{(2)}$ are independent of the permeability of the formation and the viscosity of the fluid. It turns out that the relaxation time also disappears from the first-order approximation of $\zeta$ both for slow and fast waves. The latter circumstance is discussed in Section 7 below.

Further on, using equation (55), we obtain that

$$
v^{(1)}= \pm v_{b} \sqrt{\frac{i \varepsilon}{\gamma_{1}+\gamma_{2} \gamma_{v}}+\ldots}
$$

and

$$
v^{(2)}= \pm v_{f} \sqrt{\gamma_{v}+\frac{\gamma_{1}}{\gamma_{2}}}+v_{f} V_{1} i \varepsilon+\ldots
$$

where $V_{1}$ is the first coefficient of the expansion of $V$ in the powers of $i \varepsilon$. The last two equations, in a combination with equation (63), imply 
that

$$
\begin{aligned}
k^{(1)} & = \pm \frac{1}{\tau_{D} v_{b}} \sqrt{\gamma_{1}+\gamma_{2} \gamma_{v}} \sqrt{-i \varepsilon}+\ldots \\
k^{(2)} & = \pm \frac{1}{\tau_{D} v_{f}} \frac{1}{\sqrt{\gamma_{v}+\frac{\gamma_{1}}{\gamma_{2}}}} \varepsilon+\ldots
\end{aligned}
$$

The imaginary part of $k$ must be negative, therefore, from (69) we infer that

$$
k^{(1)}=\frac{1}{\tau_{D} v_{b}} \sqrt{\gamma_{1}+\gamma_{2} \gamma_{v}} \frac{1-i}{\sqrt{2}} \sqrt{\varepsilon}+\ldots
$$

and, respectively,

$$
v^{(1)}=v_{b} \sqrt{\frac{1}{\gamma_{1}+\gamma_{2} \gamma_{v}}} \frac{1+i}{\sqrt{2}} \sqrt{\varepsilon}+\ldots
$$

It is interesting to notice that by virtue of equations (58), (48)

$$
W_{f}=-i \omega\left(\gamma_{1}-\gamma_{2} \zeta\right) U_{s}
$$

Hence, using equations (60), we get for the fast wave

$$
W_{f}^{\text {fast }}=-\varepsilon \omega \gamma_{2} \zeta_{1}^{(2)} U_{s}^{\text {fast }}+\ldots
$$

The right-hand side of the last equation is small with respect to $\varepsilon$. That means that at low frequencies, the fast wave is actually a coherent oscillation of the skeleton and the fluid. At the same time, for the slow wave we obtain a finite nonzero limit if the Darcy velocity amplitude is compared with the amplitude of the time-derivative of the displacement

$$
W_{f}^{\text {slow }}=-i \omega\left(\gamma_{1}+\gamma_{2} \gamma_{v}\right) U_{s}^{\text {slow }}+\ldots
$$

\section{BOUNDARY CONDITIONS}

In order to investigate the reflection coefficient, we need to impose boundary conditions at the interface between the media, i.e., at $x=0$. Let us consider two elastic media $M_{1}$ and $M_{2}$ occupying half-spaces separated by a plane. Their properties are characterized by the bulk densities $\varrho_{i}$ and the speeds of sound $v_{i}, i=1,2$. The medium $M_{2}$ is porous and saturated by a fluid. Therefore, this medium can be called solid skeleton and the space occupied by fluid is the pore space. We assume that the boundary between the media is impermeable for fluid flow and the permeability of medium $M_{2}$ is characterized by coefficient $\kappa$. Let us consider the propagation of a compression elastic wave in the direction orthogonal to the interface plane. Let us select a coordinate system with the $X$-axis directed orthogonally to the boundary between 
the media, such that at equilibrium the boundary is at $x=0$ and the fluid-saturated medium is at $x>0$.

Under the assumptions of Section 3, and neglecting the heterogeneities of the materials, we can assume that the displacements of the solid particles composing the media are parallel to $x$, and so is the flux of the fluid in the pore space. There is an important difference between the fluid and solid motion. The solid particles move more or less coherently near the respective equilibrium positions, whereas fluid particles move in a much more dispersed manner implied by the complexity of the pore space geometry. Only the mean volumetric flux or Darcy velocity of the moving fluid is parallel to $x$. This quantity is the result of averaging the microscopic fluid velocity field over a representative volume. In the case under consideration, such an averaging can be performed over a plane orthogonal to the direction of wave propagation, i.e., over a plane $x=$ Const $>0$.

Let us denote by $u_{1}$ and $u_{2}$ the displacements of the solid particles in media $M_{1}$ and $M_{2}$, respectively.

First, the continuity of the displacements and stresses suggests that

$$
\begin{aligned}
\left.u_{1}\right|_{x=0} & =\left.u_{2}\right|_{x=0} \\
-\left.\frac{1}{\beta_{1}} \frac{\partial u_{1}}{\partial x}\right|_{x=0} & =-\left.\frac{1}{\beta_{2}} \frac{\partial u_{2}}{\partial x}\right|_{x=0}+\left.\phi p\right|_{x=0}
\end{aligned}
$$

Zero fluid flux through the boundary implies

$$
\left.W_{f}\right|_{x=0}=0
$$

Boundary conditions (76)-(78) will be used in the next section for investigation of the reflection coefficient.

\section{REFLECTION}

Now, let us study the reflection coefficient. We have to link the sum of incidental and reflected signals in the medium $M_{1}$

$$
u_{1}=U_{1} e^{i\left(\omega t-k_{1} x\right)}+R U_{1} e^{i\left(\omega t+k_{1} x\right)}
$$

to the sum of slow and fast waves in medium $M_{2}$

$$
\begin{aligned}
p & =\frac{1}{\phi \beta_{f}} P_{0}^{s} e^{i\left(\omega t-k_{s} x\right)}+\frac{1}{\phi \beta_{f}} P_{0}^{f} e^{i\left(\omega t-k_{f} x\right)} \\
u_{2} & =U_{2}^{s} e^{i\left(\omega t-k_{s} x\right)}+U_{2}^{f} e^{i\left(\omega t-k_{f} x\right)}
\end{aligned}
$$

using boundary conditions (76), (77) and (78). We assume zero attenuation in medium $M_{1}$, therefore $k_{1}>0$ and $\omega k_{1}=v_{1}$ is the speed of sound in this medium. 
We obtain

$$
\left\{\begin{array}{ccccc}
(1+R) U_{1} & = & U_{2}^{s} & + & U_{2}^{f} \\
\frac{i k_{1}}{\beta_{1}}(1-R) U_{1} & = & \frac{i k_{2}^{s}}{\beta_{2}} U_{2}^{s} & + & \frac{i k_{2}^{f}}{\beta_{2}} U_{2}^{f} \\
0 & & & \frac{P_{0}^{f}+P_{0}^{s}}{\beta_{f}} \\
& =i \omega\left(-\gamma_{1}+\gamma_{2} \zeta^{s}\right) U_{2}^{s} & +i \omega\left(-\gamma_{1}+\gamma_{2} \zeta^{f}\right) U_{2}^{f}
\end{array}\right.
$$

Here we have utilized the first equation (48). Further on, by virtue of equation (47), we get

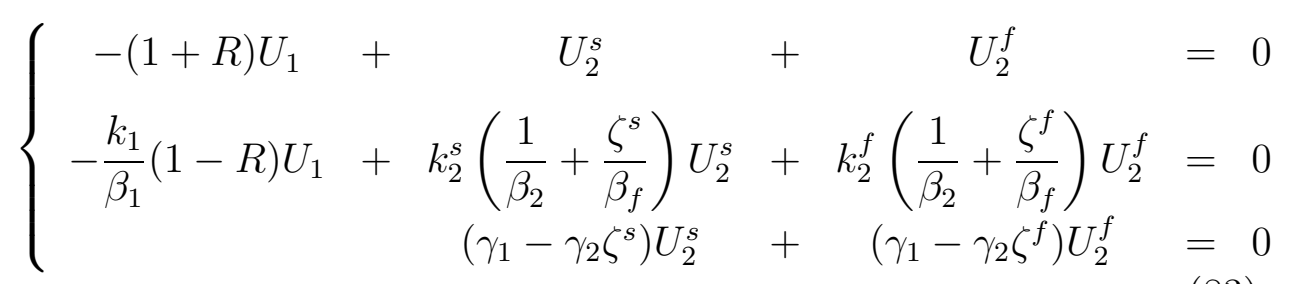

Note that in medium $M_{1}$ we have

$$
k_{1}=\frac{1}{v_{1}} \omega
$$

where $v_{1}$ is the speed of sound, i.e., a characteristic of the medium, which does not depend on the frequency.

Dividing through by $U_{1}$ and putting $Z_{1}=R, Z_{2}=U_{2}^{s} / U_{1}$, and $Z_{3}=U_{2}^{f} / U_{1}$, we obtain the following system of equations

$$
\left\{\begin{array}{ccccc}
-Z_{1}+ & Z_{2} & + & Z_{3} & = \\
\omega Z_{1}+v_{1} k_{2}^{s}\left(\frac{\beta_{1}}{\beta_{2}}+\zeta^{s} \frac{\beta_{1}}{\beta_{f}}\right) Z_{2} & + & v_{1} k_{2}^{f}\left(\frac{\beta_{1}}{\beta_{2}}+\zeta^{f} \frac{\beta_{1}}{\beta_{f}}\right) Z_{3} & = & \omega \\
\left(\gamma_{1}-\gamma_{2} \zeta^{s}\right) Z_{2} & + & \left(\gamma_{1}-\gamma_{2} \zeta^{f}\right) Z_{3} & = & 0
\end{array}\right.
$$

Hence, using equations (70) and (69) and notation (53), we can write down the system of equation (85) in the following asymptotic form

$$
\left\{\begin{array}{cccccc}
-Z_{1}+ & Z_{2} & + & Z_{3} & = & 1 \\
\sqrt{\varepsilon} Z_{1}+ & A_{22} Z_{2} & + & A_{23} \sqrt{\varepsilon} Z_{3} & = & \sqrt{\varepsilon} \\
& \left(A_{32}^{(1)}+A_{32}^{(2)} i \varepsilon\right) Z_{2}+ & A_{33} i \varepsilon Z_{3} & = & 0
\end{array}\right.
$$


The expressions for the coefficients $A_{i j}$ can be obtained from the asymptotic formulae (60), (63), (64), (70), and (71):

$$
\begin{aligned}
A_{22} & =\frac{v_{1}}{v_{b}} \sqrt{\gamma_{1}+\gamma_{2} \gamma_{v}} \gamma_{s} \frac{1-i}{\sqrt{2}} \\
A_{23} & =\frac{v_{1}}{v_{f}} \sqrt{\frac{\gamma_{2}}{\gamma_{1}+\gamma_{2} \gamma_{v}}} \gamma_{f} \\
A_{32}^{(1)} & =\gamma_{1}+\gamma_{2} \gamma_{v} \\
A_{32}^{(2)} & =-\gamma_{2} \gamma_{v} \frac{1-\gamma_{\varrho}\left(\gamma_{2} \gamma_{v}+\gamma_{1}\right)}{\gamma_{1}+\gamma_{2} \gamma_{v}} \\
A_{33} & =-\frac{\gamma_{\varrho} \gamma_{1}-\gamma_{1}+\gamma_{\varrho}}{\gamma_{1}+\gamma_{2} \gamma_{v}}
\end{aligned}
$$

Here, for brevity, we denote

$$
\gamma_{s}=\beta_{1}\left(\frac{1}{\beta_{2}}-\gamma_{v} \frac{1}{\beta_{f}}\right) \quad \text { and } \quad \gamma_{f}=\beta_{1}\left(\frac{1}{\beta_{2}}+\frac{\gamma_{1}}{\gamma_{2}} \frac{1}{\beta_{f}}\right)
$$

From the last equation (86),

$$
Z_{2}=-\frac{A_{33}}{A_{32}^{(1)}} i \varepsilon Z_{3}+\ldots
$$

This means that at low frequencies (i.e., at $\varepsilon \rightarrow 0$ ), the slow wave displacement is scaled with the velocity of fast displacement and, therefore, is one order of magnitude smaller. In other words, the slow part of the signal practically does not propagate and is mostly responsible for the reflection.

Substitution of (93) into the first two equations (86) yields

$$
\left\{\begin{array}{l}
-Z_{1}+\left(1-\frac{A_{33}}{A_{32}^{(1)}} i \varepsilon\right) Z_{3}=1 \\
\sqrt{\varepsilon} Z_{1}+\left(A_{23} \sqrt{\varepsilon}-A_{22} \frac{A_{33}}{A_{32}^{(1)}} i \varepsilon\right) Z_{3}=\sqrt{\varepsilon}
\end{array}\right.
$$

Cancelling the $\sqrt{\varepsilon}$ in the second equation (94) and dropping terms of the order higher than $\sqrt{\varepsilon}$, we obtain that

$$
Z_{3}=Z_{1}+1
$$

Consequently,

$$
Z_{1}=\frac{1-A_{23}+A_{22} \frac{A_{33}}{A_{32}^{(1)}} i \sqrt{\varepsilon}}{1+A_{23}-A_{22} \frac{A_{33}}{A_{32}^{(1)}} i \sqrt{\varepsilon}}
$$


Again, retaining only the terms of the order $\sqrt{\varepsilon}$, we finally obtain

$$
Z_{1}=\frac{1-A_{23}}{1+A_{23}}+\sqrt{2} \frac{\tilde{A}_{22} A_{33}}{A_{32}^{(1)}} \frac{1}{\left(1+A_{23}\right)^{2}}(1+i) \sqrt{\varepsilon}
$$

where

$$
\tilde{A}_{22}=\frac{v_{1}}{v_{b}} \sqrt{\gamma_{1}+\gamma_{2} \gamma_{v}} \gamma_{s}
$$

Analysis of the expression (88) yields that in practically interesting situations, the coefficient $A_{23}$ is greater than one. Therefore, the frequencyindependent component of the reflection coefficient is negative. The frequency-dependent component of the reflection has the same sign as $\tilde{A}_{33}$. The latter is positive if and only if

$$
\gamma_{\varrho}<\frac{\gamma_{1}}{1+\gamma_{1}}
$$

The right-hand side of the last inequality is approximately equal to 0.5. Hence, roughly speaking, $\tilde{A}_{33}$ is positive when the fluid density is at least twice less than the bulk density of the saturated medium. In such a case the maximum of the absolute value of the reflection coefficient is attained at $\varepsilon=0$. At the same time, for dense fluids, the first-order term of the asymptotic expansion, which is proportional to the square root of $\varepsilon$, may vanish and the first frequency-dependent term will be linear. In this case, the tortuosity coefficient becomes an important factor.

In the original variables (51), equation (97) takes on the form

$$
R=\frac{1-A_{23}}{1+A_{23}}+\sqrt{2} \frac{\tilde{A}_{22} A_{33}}{A_{32}^{(1)}} \frac{1}{\left(1+A_{23}\right)^{2}}(1+i) \sqrt{\frac{\kappa \varrho_{b}}{\eta} \omega}
$$

Note that the last equation relates the reflectivity to the frequency through the factor of $\tau_{D}=\frac{\kappa \varrho_{b}}{\eta}$ having the dimension of time. It involves a property of the rock, the permeability coefficient, a property of the fluid, the viscosity, and a property of the coupled fluid-rock system, the bulk density. The latter implies that the density of the fluid only partially affects the reflectivity. This fact has been confirmed in laboratory experiments [20], where it was observed that the dependence of the reflected signal on fluid viscosity plays major role. Note that the frequency scaling proposed here is similar to but not the same as the scaling introduced in [18].

\section{The ROLE OF RELAXATION TIME AND TORTUOSITY}

The asymptotic calculations presented above show that the dimensionless parameter $\theta$ related to both relaxation time and tortuosity 
factor disappears from the first-order terms. However, if $\theta$ is a large number, then some expansions obtained in Sections 4 and 6 have to be reviewed. Practically, the range of the frequencies where the analysis is technically feasible is limited by the specifications of the available tools. Therefore, it may happen that within the range of frequencies available for analysis the product $\theta \varepsilon$ is not negligibly small. Therefore, the theoretical passage to the limit as $\varepsilon \rightarrow 0$ should be replaced with analysis at some intermediate finite values of $\varepsilon$. In such a case, the asymptotic analysis is performed differently. In this section, we consider two such situations.

First, let us assume that within the range of available frequencies, the parameter $\varepsilon \theta$ is of the order of one. In original variables, this condition is equivalent to

$$
\omega \sim \frac{1}{\tau}
$$

We have to return back to the quadratic equation (56) for the parameter $\zeta$. Regrouping the coefficients and dividing through by $1+i \theta \varepsilon$, we rewrite this equation in the form

$$
\left(A_{0}+A_{1}^{\theta} i \varepsilon\right) \zeta^{2}+\left(B_{0}+B_{1}^{\theta} i \varepsilon\right) \zeta+C_{0}+C_{1}^{\theta} i \varepsilon=0
$$

where the coefficients with the zero index are the same as in equation (61), and

$$
\begin{aligned}
A_{1}^{\theta} & =-\frac{\gamma_{2} \gamma_{\varrho}}{1+i \theta \varepsilon} \\
B_{1}^{\theta} & =\frac{-1+\gamma_{\varrho}\left(1+\gamma_{1}\right)}{1+i \theta \varepsilon} \\
C_{1}^{\theta} & =\frac{\gamma_{v} \gamma_{\varrho}}{1+i \theta \varepsilon}
\end{aligned}
$$

Hence, the frequency-independent zero-terms of asymptotic expansions of the solutions $\zeta$ are the same as in equation (58). To calculate the first order coefficients, we note that formally the coefficients (103) are equal to the respective coefficients in equations (61) evaluated at $\tau=0$ and divided by $1+i \theta \varepsilon$. This fact an conjunction with the observation that the asymptotic expansion of the reflection coefficient (100) does not depend on $\tau$ significantly simplifies the calculations. Indeed, for the first-order coefficients of asymptotic expansion for $\zeta$ we can reuse equations (63) and (64) if we put there $\tau=0$ and multiply the righthand sides by an additional factor of $\frac{1}{1+i \theta \varepsilon}$. Clearly, the calculations for the first order terms of expansions of $v$ and $k$ can be carried out in a similar manner. The final result is that the reflection coefficient in 
the asymptotic expression (100) takes on the form

$$
R=\frac{1-A_{23}}{1+A_{23}}+2 \frac{A_{22} A_{33}}{A_{32}^{(1)}} \frac{1}{\left(1+A_{23}\right)^{2}} \sqrt{i-\theta \varepsilon} \sqrt{\frac{\kappa \varrho_{b}}{\eta} \omega}
$$

Thus, in a case where $\tau \omega=O(1)$, the relaxation time and tortuosity affect both the amplitude and the phase shift of the reflected signal.

Now, let us consider another extreme situation where $\theta \gg 1$, so that after a division of equation (56) by $\theta$ all terms with $\theta$ in the denominator can be neglected. In such a case, we obtain a quadratic equation

$$
i \varepsilon\left(A_{0} \zeta^{2}+B_{0} \zeta+C_{0}\right)=0
$$

The latter implies that the dependence on the frequency of $\zeta$ and, therefore, of the reflection coefficient as well, vanishes. This conclusion can be interpreted in the following way: at a very large relaxation time (or, equivalently, at a very large tortuosity), the inertial term in equation (38) makes the dissipation term on the right-hand side unimportant. Consequently, the fluid-saturated medium acts as an elastic composite medium and we arrive at a classical elastic wave reflection independent of the frequency.

\section{Conclusions}

Equations of elastic waves propagation in fluid-saturated porous media have been obtained form the basic principles of hydrology. It has been demonstrated, that under different assumptions, these equations can be reduced either to Biot's poroelasticity model or to the pressure diffusion equation. The low-frequency asymptotic behavior of the reflection of a planar seismic signal from an interface between an elastic medium and fluid-saturated porous medium has been investigated. It has been obtained that the frequency-dependent component of the reflection coefficient is scaled with the square root of the dimensionless including the frequency and the reservoir fluid mobility. The dependence of this characteristic time on the flow properties of the medium and the fluid has been investigated.

\section{ACKNOWLEDGMENTS}

This work has been performed at Lawrence Berkeley National Laboratory of the U.S. Department of Energy under Contract No. DEAC03-76SF00098, the University of California at Berkeley, and at the University of Houston. The authors are thankful to Dr. Steven Pride of Lawrence Berkeley National Laboratory (LBNL) for fruitful discussions and to Prof. G. I. Barenblatt of University of California at Berkeley 
and LBNL for critical remarks. Both helped to significantly improve the presentation.

\section{REFERENCES}

1. M. G. Alishaev, Proceedings of Moscow Pedagogy Institute, 1974, pp. 166-174.

2. M. G. Alishaev and A. Kh. Mirzadzhanzadeh, On retardation phenomena in filtration theory (in Russian), Neft i Gaz (1975), no. 6, 71-74.

3. G. I. Barenblatt, V. M. Entov, and V. M. Ryzhik, Theory of fluid flows through natural rocks, Kluwer Academic Publishers, Dordrecht, 1990.

4. G. I. Barenblatt and A. P. Vinnichenko, Non-equilibrium seepage of immiscible fluids, Advances in Mechanics 3 (1980), no. 3, 35-50.

5. G.I. Barenblatt, Filtration of two nonmixing fluids in a homogeneous porous medium, Soviet Academy Izvestia. Mechanics of Gas and Fluids (1971), no. 5, 857-864.

6. J. Bear, Dynamics of fluids in porous media, Elsevier, N.Y., 1972.

7. M. A. Biot, Theory of propagation of elastic waves in a fluid-saturated porous solid. I. low-frequency range, Journal of the Acoustical Society of America 28 (1956), no. 2, 168-178.

8. Theory of propagation of elastic waves in a fluid-saturated porous solid. II. higher frequency range, Journal of the Acoustical Society of America 28 (1956), no. 2, 179-191.

9. Mechanics of deformation and acoustic propagation in porous media, Journal of Applied Physics 33 (1962), no. 4, 1482-1498.

10. J. P. Castagna, S. Sun, and R. W. Siegfried, Instantanous spectral analysis: Detection of low-frequency shadows associated with hydrocarbons, The Leading Edge (2003), 120-127.

11. H. Darcy, Les fontaines de la ville de Dijon, Victor Dalmont, Paris, 1856.

12. A. I. M. Denneman, G. G. Drijkoningen, D. M. J. Smeulders, and K. Wapenaar, Reflection and transmission of waves at a fluid/porous-medium interface, Geophysics 67 (2002), no. 1, 282-291.

13. O. Yu. Dinariev and O. V. Nikolaev, On relaxation processes in low-permeability porous materials, Eng. Phys. Journal 55 (1990), no. 1, 78-82.

14. N. C. Dutta and H. Ode, Attenuation and dispersion of compressional-waves in fluid-filled rocks with partial gas saturation (White model) - Part I: Biot theory, Geophysics 44 (1979), no. 11, 1777-1788.

15. _ Seismic reflections from a gas-water contact, Geophysics 48 (1983), no. $02,148-162$.

16. J. Frenkel, On the theory of seismic snf seismoelectric phenomena in a mois soil, Journal of Physics 8 (1944), no. 4, 230-241.

17. F. Gassmann, Über die Elastizität poröser Medien, Vierteljahrscrift Naturforsch Ges. Zürich 96 (1951), 1-23.

18. J. Geertsma and D. C. Smit, Some aspects of elastic wave propagation in fluidsaturated porous solids, Geophysics 26 (1961), no. 2, 169-181.

19. G. M. Goloshubin and A.V. Bakulin, Seismic reflectivity of a thin porous fluid-saturated layer versus frequency, 68th SEG Meeting (New Orleans), 1998, pp. 976-979.

20. G. M. Goloshubin and V. A. Korneev, Seismic low-frequency effects from fluidsaturated reservoir, SEG Meeting (Calgary), 2000. 
21. M. King Hubbert, The theory of ground-water motion, Journal of Geology 48 (1940), 785-943.

22. D_ Darcy's law and the field equations of the flow of underground fluids, Trans. AIME 207 (1956), no. 7, 222-239.

23. V. A. Korneev, G. M. Goloshubin, T. M. Daley, and D. B. Silin, Seismic lowfrequency effects in monitoring of fluid-saturated reservoirs, Geophysics (2004), to appear.

24. L. D. Landau and E. M. Lifschitz, Fluid mechanics, Series in advanced physics, vol. 6, Addison-Wesley, Reading, MA, 1959.

25. C. S. Matthews and D. G. Russell, Pressure buildup and flow tests in wells, Monograph Series, Society of Petroleum Engineers, New York, 1967.

26. Yu. M. Molokovich, Problems of filtration theory and mechanics of oil recovery improvement, Nauka, Moscow, 1987.

27. Yu. M. Molokovich, N. N. Neprimerov, B. I. Pikuza, and A. V. Shtanin, Relaxational filtration (in Russian), Kazan University, Kazan, 1980.

28. L. N. Molotkov, On coefficients of pore tortuosity in an effenctive Biot model (in russian), Transactions of St.-Petersburg branch of Steklov mathematical institute 257 (1999), 157-164.

29. M. Muskat, The flow of homogeneous fluids in porous media, McGrow-Hill, 1937.

30. T. W. Patzek, Verification of a complete pore network simulator of drainage and imbibition, SPE Journal 6 (2001), no. 2, 144-156.

31. P. Y. Polubarinova-Kochina, Theory of groundwater movement, Princeton University Press, Princeton, N. J., 1962.

32. S. R. Pride, J. M. Harris, D. L. Johnson, A. Mateeva, K. T. Nihei, R. L. Noeack, J. W. Rector, H. Spelzler, R. Wu, T. Yamomoto, J. G. Berryman, and M. Fehler, Permeability dependence of seismic amplitudes, The Leading Edge (2003), 518-525.

33. Steven R. Pride and James G. Berryman, Linear dynamics of double-porosity dual-permeability materials. I. Governing equations and acoustic attenuation, Physical Review E 68 (2003), no. 3, 036603.

34. _ Linear dynamics of double-porosity dual-permeability materials. II. Fluid transport equations, Physical Review E 68 (2003), no. 3, 036604.

35. J. E. Santos, J. M. Corbero, C. L. Ravazzoli, and J. L. Hensley, Reflection and transmission coefficients in fluid-saturated porous media, Journal of Acoustical Society of Amerika 91 (1992), no. 1, 1911-1923.

36. D. B. Silin, J. Guodong, and T. W. Patzek, Paper spe 84296, robust determination of the pore space morphology in sedimentary rocks, SPE Annual Technical Conference and Exhibition. 5-8 October 2003. (Denver, CO), SPE, 2003.

37. D. B. Silin and T. W. Patzek, On Barenblatt's model of spontaneous countercurrent imbibition, Transport in Porous Media 54 (2004), no. 3, 297-322.

38. Herbert F. Wang, Theory of linear poroelasticity, Princeton Series in Geophysics, Princeton University Press, Princeton, NJ, 2000. 
Lawrence Berkeley National Laboratory, 1 Cyclotron Road, MS 90-1116, Berkeley, CA 94720, USA

E-mail address: DSilin@lbl.gov

Lawrence Berkeley National Laboratory, 1 Cyclotron Road, MS 90-1116, Berkeley, CA 94720, USA

Department of Geosciences, University of Houston, Houston, TX 77204

Lawrence Berkeley National Laboratory, 1 Cyclotron Road, MS 90-1116, Berkeley, CA 94720, USA 